\title{
Parto en la adolescente
}

\author{
Luis Vicente López Avila*; Jacinto Sánchez Angarita**
}

RESUMEN: Durante el período comprendido entre el primero de enero a 31 de diciembre de 1987, se realizó un estudio de casos controles en el Departamento de Ginecología y Obstetricia, de la Facultad de Medicina de la Universidad Nacional, en el Instituto Materno Infantil de Bogotá, con el fin de conocer el comportamiento del parto en las adolescentes frente a un grupo de mayor edad en iguales condiciones socio-económicas. Durante este período se atendieron 8.692 partos, de los cuales 728 fueron en adolescentes (8.37\%).

Para efectos de este estudio se eligieron al azar 200 adolescentes, entre 12 a 17 años y sus correspondientes controles, primigestantes de 20 y 24 años de edad.

Se encontraron los siguientes resultados:

En relación con el estado civil se encontró que la mayoría de población era soltera y sin unión estable, siendo la unión libre más frecuente en los controles. Los factores de riesgo estadísticamente mayores en las adolescentes fueron: Preeclampsia, anemia, estrechez pélvica, parto instrumentado, menor peso en el neonato.

Se distribuyeron por igual los siguientes factores de riesgos: la infección de vías urinarias, la cual es alta en ambos grupos, el peso del neonato menor de 2.000 gr. en ambos grupos $(4.5 \%)$, lo mismo que la talla de los neonatos y las malaformaciones congénitas.

Riesgos como el estado del recién nacido, calificación de Apgar menor de 7 fue mayor en los controles, lo mismo que las complicaciones del puerperio inmediato.

Además un mayor número de cesáreas, partos en pelvis, RPM, complicaciones del puerperio inmediato, sin que fueran estadísticamente significativos.

PALABRAS CLAVES: Adolescentes Parto.

SUMMARY: During the period between January 1th of 1987 and the 31th of December of the same year, it was made an prospectiva study in cases control of adolescents deliverys in contrast with a group of women of the mild age.

These study was preformed in the Institute Materno Infantil of the Medicine Faculty in the National University of Colombia.

The total of cases as of 8692 deliverys of wich, 728 was in adolescents mothers $(8.37 \%)$.

In this particular study wend selected 200 adolescents between 12 and 17 years old and the correspondent cases control between $20-24$ years old.

The result were as follow:

In relation with the civil status was found that mayority of the population were single and with out stable relation ship in contrast with the control cases. The mayor risk in adolescents were: Preeclamsy, anemia, instrumental delivery, low weyth at birth, etc.

The following riks were egual in the both groups: Infections in the urone tract, the duration in the delivery of 9; hours like averaje in both groups. The weigth of the newborn below $2000 \mathrm{gr}$ in both grops (4.5\%) the malformations and the length of the newborns was the sanse in both groups. Risks, like apgar calificaztion below 7 , and inmediatly complications of puerperium were more important in the control cases.

We found none cesarean section, distaciz deliveries, RPM in the control group.

KEY WORDS: Adolescents Deliverys.

\section{Introducción}

La atención de la adolescente en los países en vía de desarrollo tiene interés muy especial por la frecuencia con que se inicia a temprana edad la reproducción. En nuestro país una gran mayoría de la población es adolescente por lo cual se hace de vital importancia el estudio del desenlace de la gestación en ésta.

Se cita frecuentemente en la literatura mundial algunos indicadores de riesgo que se hallan aumentados en la adoles-

\footnotetext{
* Profesor Asociado - Dpto. de Ginecología y Obstetricia - Facultad de Medicina. Universidad Nacional.

** Profesor Asistente - Dpto. de Ginecología y Obstetricia - Facultad de Medicina. Universidad Nacional.
}

cente, como son: La toxemia, la anemia, el parto prolongado, la pelvis estrecha, la prematurez, el bajo peso al nacer, el aumento de malformaciones y la mortalidad neonatal. Sin embargo, hay otros investigadores para los cuales las complicaciones del parto y puerperio en la adolescente tiene una incidencia muy similar de la observada en los grupos de más edad, pero con iguales condiciones socio-económicas. Por lo tanto el factor edad en sí, no es el responsable de las complicaciones $(7-8)$.

Con el fin de establecer el comportamiento en el parto y puerperio de la población adolescente que asiste al Instituto Materno Infantil de Bogotá, se llevó a cabo un estudio de casos y controles. 


\section{Material y métodos}

Durante el tiempo comprendido entre enero $1^{\circ}$ al 31 de diciembre de 1987, período del estudio, se atendieron en este Hopsital 8.692 partos, de los cuales 728 fueron de adolescentes $(8.37 \%)$. La edad de las adolescentes parturientas estuvo entre 12 a 17 años cumplidos en el momento del parto.

Se distribuyeron así:

\begin{tabular}{|lcr|}
\hline Edad & No. Pacientes & $\%$ \\
\hline 17 años & 343 & 47.11 \\
16 años & 220 & 30.21 \\
15 años & 122 & 16.75 \\
14 años & 34 & 4.67 \\
13 años & 7 & 0.96 \\
12 años & 2 & 0.27 \\
\hline
\end{tabular}

El número de cesáreas durante el mismo tiempo fue de $2.002(23.02 \%)$. En las adolescentes se practicaron 137 cesáreas $(18.82 \%)$ distribuidas así:

PARTO POR CESAREA EN LAS ADOLESCENTES

\begin{tabular}{|lcrccc|}
\hline Edad & No. de partos & \% & No. de cesáreas & $\%$ \\
\hline 17 años & 343 & 47.11 & 57 & 16.61 \\
16 años & 220 & 30.21 & 47 & 21.36 \\
15 años & 122 & 16.75 & 21 & 17.21 \\
14 años & 34 & 4.67 & 9 & 26.47 \\
13 años & 7 & 0.96 & 3 & 42.85 \\
12 años & 2 & 0.27 & 0 & 0.0 \\
\hline
\end{tabular}

Para efecto de este estudio se eligió una muestra al azar de 200 adolescentes y sus correspondientes controles de la población primigestantes que asistieron al I.M.I. durante el período de estudio y tenían una edad comprendida entre los 20 a 24 años.

La elección se hizo así:

De las 728 adolescentes menores de 17 años que asistieron durante el año de estudio se eligió la primera adolescente que llegó al Hopsital y luego la primera primigestante que llegó inmediatamente después, con edad de 20 a 24 años, y luego la que le sigue inmediatamente después, y así sucesivamente hasta completar las 200 primigestantes de 20 a 24 años, que fue el número de éstas que llegó durante el período de estudio.

\section{Resultados}

Se analizó el estado civil de la población y los resultados fueron así:

Tabla 1

ESTADO CIVIL

\begin{tabular}{|lcccc|}
\hline & \multicolumn{3}{c}{ Adolescentes } & Controles \\
& No. & $\%$ & No. & $\%$ \\
\hline Solteras & 152 & 76 & 124 & 62 \\
Unión libre & 14 & 7 & 52 & 26 \\
Casada & 34 & 17 & 24 & 12 \\
\hline
\end{tabular}

De la anterior tabla se deduce que la gran mayoría de nuestras pacientes son solteras y que la unión libre es más frecuente en el grupo control, considerando éste como la convivencia permanente por más de un año.

En relación con el control prenatal, se considera que asistió cuando tuvo dos o más controles, y entonces tenemos:

Tabla 2

CONTROL PRENATAL

\begin{tabular}{|lcccc|}
\hline & \multicolumn{2}{c|}{ Adolescentes } & \multicolumn{2}{c|}{ Controles } \\
& No. & $\%$ & No. & $\%$ \\
\hline Asistió & 117 & 58.5 & 130 & 65 \\
No asistió & 83 & 41.5 & 70 & 35 \\
\hline
\end{tabular}

Que se observa una mayor frecuencia en el grupo control, no existe diferencia estadísticamente significativa.

En relación con el parámetro complicaciones del prenatal tenemos lo siguiente:

Tabla 3

COMPLICACIONES DEL PRENATAL

\begin{tabular}{|c|c|c|c|c|}
\hline \multirow[t]{2}{*}{ Patología } & \multicolumn{2}{|c|}{ Adolescentes } & \multicolumn{2}{|c|}{ Controles } \\
\hline & No. & $\%$ & No. & $\%$ \\
\hline Inf. Vías urinarias & 25 & 12.5 & 30 & 15.0 \\
\hline Pre-Eclampsia & 29 & 14.5 & 21 & 10.5 \\
\hline Anemia & 8 & 4.0 & 1 & 1.0 \\
\hline Enf. Venérea & 7 & 3.5 & 4 & 2.0 \\
\hline Amenaza & & & & \\
\hline de part. pret. & 6 & 3.0 & 6 & 3.0 \\
\hline Muerte fetal & 3 & 1.5 & 2. & 1.0 \\
\hline
\end{tabular}

En la tabla anterior podemos observar que fue notoria la diferencia entre las adolescentes y los controles, en lo que se refiere a la pre-eclampsia y el estado anémico de la paciente en el prenatal, presentándose una diferencia estadísticamente significativa.

Además podemos observar una frecuencia notoria de infección en las vías urinarias en ambos grupos.

$\mathrm{Si}$ consideramos el estado de la paciente en su ingreso tenemos:

Tabla 4

EXAMEN MEDICO DE ADMISION

\begin{tabular}{|lrrrr|}
\hline Parámetros & \multicolumn{2}{c}{ Adolescentes } & \multicolumn{2}{c|}{ Controles } \\
& No. & \% & No. & $\%$ \\
\hline En trabajo de parto & 171 & 85.5 & 163 & 81.5 \\
Sin trabajo de parto & 29 & 14.5 & 37 & 18.5 \\
Conscientes & 196 & 98.0 & 197 & 98.5 \\
Inconscientes & 4 & 2.0 & 3 & 1.5 \\
Edemas & 22 & 11.0 & 20 & 10.0 \\
Sin edemas & 178 & 89.0 & 180 & 90.0 \\
Con anemia & 17 & 8.5 & 10 & 5.0 \\
Sin anemia & 183 & 91.5 & 190 & 95.0 \\
Fetos vivos & 199 & 99.5 & 197 & 98.5 \\
Fetos muertos & 1 & 0.5 & 3 & 1.5 \\
\hline \multicolumn{2}{c}{$\mathrm{P}<0.044$} & & \\
\hline
\end{tabular}

En términos generales puede decirse que no hubo diferencia estadísticamente significativa y que las dos poblaciones se comportaron uniformemente igual exceptuando el estado anémico de las madres en el cual se anota un número mayor en las adolescentes sin que sea estadísticamente significativa la diferencia, en relación con el estado de vitalidad fetal fue notoria la diferencia en favor de las adolescentes $(\mathrm{P}<.044)$.

En relación con las cifras tensionales tenemos que la distribución de la población fue la siguiente:

Tabla 5

NIVELES TENSIONALES

\begin{tabular}{|l|rrrr|}
\hline Tensión arterial & \multicolumn{2}{|c|}{ Adolescentes } & \multicolumn{2}{c|}{ Controles } \\
& No. & $\%$ & No. & $\%$ \\
\hline$<140 / 90$ & 174 & 87 & 167 & 83.5 \\
$140 / 90$ a $160 / 110$ & 22 & 11 & 31 & 15.5 \\
$>160 / 110$ & 4 & 2 & 2 & 1.0 \\
\hline
\end{tabular}

Como se puede ver en este parámetro no hubo diferencia estadísticamente significativa, el comportamiento de ambas poblaciones fue semejante.

En relación con el parámetro ruptura prematura de membranas el comportamiento de las dos poblaciones fue el siguiente: 
Tabla 6

RUPTURA PREMATURA DE MEMBRANAS

\begin{tabular}{|lcccc|}
\hline & \multicolumn{2}{c}{ Adolescentes } & \multicolumn{2}{c|}{ Controles } \\
& No. & \% & No. & \% \\
\hline Presentaron RPM & 35 & 17.5 & 39 & 19.5 \\
No presentaron RPM & 165 & 82.5 & 161 & 80.5 \\
\hline
\end{tabular}

En relación con la ruptura prematura de membranas aunque se observa un ligero aumento en el grupo de control, este aumento no es estadísticamente significativo.

En relación con el tipo de parto tenemos los datos siguientes:

Tabla 7

TIPO DE PARTO

\begin{tabular}{|lrlll|}
\hline & \multicolumn{2}{c}{ Adolescentes } & \multicolumn{2}{c|}{ Controles } \\
& No. & \% & No. & \% \\
\hline Parto espontáneo & 105 & 52.5 & 103 & 51.5 \\
Parto instrumentado & 53 & 26.5 & 47 & 23.5 \\
Parto por cesárea & 42 & 21.0 & 50 & 25.0 \\
\hline
\end{tabular}

En relación con el tipo de parto no se observa diferencia estadísticamente significativa.

En relación con las complicaciones presentes durante el parto tenemos:

Tabla 8

COMPLICACIONES DEL PARTO

\begin{tabular}{|llclr|}
\hline Complicaciones & \multicolumn{2}{l}{ Adolescentes } & \multicolumn{2}{l|}{ Controles } \\
& No. & \% & No. & \% \\
\hline Ninguna & 158 & 79.0 & 157 & 78.5 \\
Trab. prolongado & 10 & 5.0 & 7 & 3.5 \\
Desp. cefalopélvica & 4 & 2.0 & 6 & 3.0 \\
Placenta previa & 2 & 1.0 & 1 & 0.5 \\
Estrechez pélvica & 8 & 4.0 & 4 & 2.0 \\
Abruptio placentae & 1 & 0.5 & 2 & 1.0 \\
Hemorragia postparto & 3 & 1.5 & 2 & 1.0 \\
Convulsiones intra parto & 2 & 1.0 & 1 & 0.5 \\
Parto en pelvis & 11 & 5.5 & 19 & 19.5 \\
Otros & 1 & 0.5 & 1 & 0.5 \\
\hline
\end{tabular}

Como se puede ver en la tabla anterior no hubo diferencia estadísticamente significativa en la muestra examinada, sin embargo, podemos observar que los partos en pelvis fueron más frecuentes en el grupo control que en las adolescentes, y la estrechez pélvica más frecuente en las adolescentes.

Otro parámetro observado fue la duración del parto y en el cual encontramos los siguientes datos:

Tabla 9

DURACION DEL PARTO

\begin{tabular}{|llrrr|}
\hline No. Horas & \multicolumn{2}{c|}{ Adolescentes } & \multicolumn{2}{c|}{ Controles } \\
& No. & \% & No. & \% \\
\hline$<4$ & 34 & 17.0 & 35 & 17.5 \\
5 a 8 & 64 & 32.0 & 72 & 36.0 \\
9 a 12 & 57 & 28.5 & 54 & 27.0 \\
13 a 16 & 27 & 13.5 & 25 & 12.5 \\
> 17 & 18 & 9.0 & 14 & 7.0 \\
\hline
\end{tabular}

La cual no muestra diferencia entre los dos grupos.

En relación con la calificación del Apgar obtuvimos los siguientes datos:

Tabla 10

CLASIFICACION DE APGAR (A LOS 5 MINUTOS)

\begin{tabular}{|lrrrr|}
\hline Clasificación & \multicolumn{2}{c|}{ Adolescentes } & \multicolumn{2}{c|}{ Controles } \\
& No. & \% & No. & \% \\
\hline$>8$ & 190 & 95.0 & 183 & 91.5 \\
5 a 7 & 6 & 3.0 & 14 & 7.0 \\
$<4$ & 4 & 2.0 & 3 & 1.5 \\
\hline \multicolumn{5}{c}{$\mathrm{P}<0.0074$}
\end{tabular}

En relación con la tabla 10 se halla una diferencia estadísticamente significativa que favorece a los adolescentes, ya que los recién nacidos de ellas presentaron calificación de Apgar más alto. Esto es, que nacieron en mejores condiciones en relación con los controles.

En relación con la talla del recién nacido los datos registrados fueron los siguientes:

Tabla 11

TALLA DEL RECIEN NACIDO

\begin{tabular}{|lrrrr|}
\hline & \multicolumn{2}{c}{ Adolescentes } & \multicolumn{2}{c|}{ Controles } \\
Centímetros & No. & $\%$ & No. & $\%$ \\
\hline$<45$ & 26 & 13.0 & 22 & 11.0 \\
46 a 52 & 170 & 85.0 & 172 & 86.0 \\
$>53$ & 4 & 2.0 & 6 & 3.0 \\
\hline
\end{tabular}

Como se puede ver en la tabla anterior, la población de recién nacidos se distribuyó en forma semejante en ambos grupos sin que se notara diferencia estadísticamente significativa.

En relación con el peso del recién nacido los datos observados fueron los siguientes:

Tabla 12

PESO DEL RECIEN NACIDO

\begin{tabular}{|lcrrr|}
\hline & \multicolumn{2}{c|}{ Adolescentes } & \multicolumn{2}{c|}{ Controles } \\
Gramos & No. & \multicolumn{1}{c|}{$\%$} & \multicolumn{1}{c|}{ No. } & \multicolumn{1}{c|}{$\%$} \\
\hline$<1999$ & 9 & 4.5 & 9 & 4.5 \\
2000 a 2999 & 93 & 46.5 & 88 & 44.0 \\
3000 a 3999 & 97 & 48.5 & 99 & 49.5 \\
$>4000$ & 1 & 0.5 & 4 & 2.0 \\
\hline
\end{tabular}

En relación con este parámetro podemos observar que los pesos menores de $2.000 \mathrm{gr}$ fueron semejantes en ambos grupos sin embargo, los pesos mayores de $3.000 \mathrm{gr}$. fueron estadísticamente significativa. $(\mathrm{P}<.0022)$ en favor de los controles.

En relación con las malformaciones del recién nacido tenemos que los resultados fueron los siguientes:

Tabla 13

MALFORMACIONES DEL RECIEN NACIDO

\begin{tabular}{|lrrrr|}
\hline & Adolescentes & \multicolumn{3}{c|}{ Controles } \\
& No. & \% & No. & $\%$ \\
\hline No hubo & 196 & 98.0 & 196 & 98.0 \\
Menores & 2 & 1.0 & 3 & 1.5 \\
Mayores & 2 & 1.0 & 1 & 0.5 \\
\hline
\end{tabular}

En relación con este parámetro el comportamiento fue muy semejante y no se observó diferencia estadísticamente significativa entre los dos grupos.

En relación con las complicaciones del puerperio inmediato la observación nos muestra los siguientes datos:

Tabla 14

COMPLICACIONES DEL PUERPERIO INMEDIATO

\begin{tabular}{|lrrrr|}
\hline & \multicolumn{2}{c}{ Adolescentes } & \multicolumn{2}{c|}{ Controles } \\
& No. & \% & No. & $\%$ \\
\hline No hubo complicaciones & 182 & 81.0 & 176 & 88.0 \\
Atonía uterina & 0 & 0.0 & 3 & 1.5 \\
Shock hipovolémico & 2 & 1.0 & 1 & 0.5 \\
Desgarro perineal & 12 & 6.0 & 18 & 9.5 \\
Otros & 4 & 2.0 & 2 & 1.0 \\
\hline
\end{tabular}

En relación con esta tabla podemos decir que no hubo diferencia estadísticamente significativa, sin embargo se 
observa una ligera diferencia en relación con la atonía y los desgarros perineales que favorecen a las adolescentes.

En relación con las complicaciones del neonato tenemos los siguientes resultados:

Tabla 15

COMPLICACIONES DEL NEONATO

\begin{tabular}{|lrrrr|}
\hline & \multicolumn{2}{c|}{ Adolescentes } & \multicolumn{2}{c|}{ Controles } \\
& No. & \multicolumn{1}{c|}{$\%$} & \multicolumn{1}{c|}{ No. } & $\%$ \\
\hline Ninguna & 176 & 88.0 & 173 & 86.5 \\
Prematuros & 14 & 7.0 & 11 & 5.5 \\
Sind. Dific. Respir. & 2 & 1.0 & 3 & 1.5 \\
Infección & 4 & 2.0 & 5 & 2.5 \\
Hemorragia Int. Cereb. & 0 & 0.0 & 2 & 1.0 \\
Otras & 4 & 2.0 & 6 & 3.0 \\
\hline
\end{tabular}

En relación con las complicaciones del neonato se observó un ligero aumento en los controles sin que éste fuera estadísticamente significativo.

\section{Complicaciones del puerperio}

Consideramos las complicaciones del puerperio en los primeros 8 días y obtuvimos los siguientes resultados:

Tabla 16

COMPLICACIONES DEL PUERPERIO

\begin{tabular}{|c|c|c|c|c|}
\hline \multirow[b]{2}{*}{ Tipo } & \multicolumn{2}{|c|}{ Adolescentes } & \multicolumn{2}{|c|}{ Controles } \\
\hline & No. & $\%$ & No. & $\%$ \\
\hline No hubo & 191 & 95.5 & 185 & 92.5 \\
\hline Infección genital & 6 & 3.0 & 12 & 6.0 \\
\hline Infección vías urinarias & 0 & 0.0 & 1 & 0.5 \\
\hline Dehiscencia de suturas & 3 & 1.5 & 1 & 0.5 \\
\hline Otras & 0 & 0.0 & 1 & 0.5 \\
\hline
\end{tabular}

En relación con las complicaciones del puerperio a los 8 días podemos establecer que se presentó con más frecuencia en los controles, sobre todo en lo que hizo referencia a las infecciones genitales.

\section{Discusión}

Los que se han preocupado por la adolescente embarazada, algunas veces consideran el parto y puerperio como de alto riesgo y otras veces como de un riesgo no mayor al de la población con igual nivel socio-económico.

Al analizar nuestros resultados tenemos que en relación con los partos por cesárea un $18.85 \%$ para las adolescentes y un $23.02 \%$ para la población en general que asistió al Instituto durante el mismo período del estudio. Pero si distribuimos las adolescentes por grupos de edades, tenemos que las menores de 15 años tuvieron un porcentaje sensiblemente mayor, las de 14 años $(26.47 \%), 13$ años $(42.85 \%)$ en cambio las mayores de 15 años mostraron un porcentaje menor, así, (17.21\%) para las de 15 años, $(21.36 \%)$ para las de 16 años y $(16.61 \%)$ para las de 17 años. Lo cual nos permite decir que existe un riesgo aumentado de cesáreas para las adolescentes menores de 15 años en cambio para las de 15 a 17 es menor.

En relación con el estado civil de nuestra población podemos decir que en su gran mayoría son solteras tanto la población de adolescentes como los controles, (62\%) y
(76\%). No hubo diferencia estadísticamente significativa entre los dos grupos (Tabla 2).

Cuando consideramos el prenatal observamos que la asistencia fue semejante en ambos grupos, pero si consideramos las complicaciones (Tabla 3 ), tenemos que las adolescentes presentaron un riesgo más alto en cuanto a enfermedades vénereas, anemia (consideración ésta como hemoglobina menor de 10 grs. y hematocrito de 33), y preeclampsia, diferencia estadísticamente significativa $(\mathrm{P}>0.053)$.

En relación con el estado de la paciente en el momento del ingreso, se analizaron los siguientes parámetros: estado de conciencia, edemas, estado tensional, ruptura prematura de membranas, viabilidad fetal y no hubo diferencia estadísticamente significativa.

En relación con el tipo y vía de parto, se observó un mayor porcentaje de parto instrumentado en las adolescentes, pero a pesar de lo que se pudiera esperar, el número de cesáreas fue mayor en los controles, sin diferencia estadística significativa.

Cuando observamos las complicaciones del parto y analizamos los siguientes parámetros: trabajo prolongado, desproporción cefalo-pélvica, estrechez pélvica, placenta previa, abruptio placentae, hemorragia postparto, y parto en pelvis no se observa diferencia estadísticamente significativa en los dos grupos, pero se anota un porcentaje ligeramente mayor de estrechez pélvica para las adolescentes $(8 \%$ y $4 \%$ ) ver tabla (8), en relación con la presentación de pelvis fue mayor para los controles $(5.5 \%$ y $9.5 \%)$.

En relación con la duración del parto no hubo diferencia entre un grupo y el otro. El promedio fue de 9 horas en los dos grupos.

En relación con el estado del niño, calificación de Apgar a los 5 minutos, se observó una diferencia estadísticamente significativa a favor de las adolescentes $(\mathrm{P}<.0074)$. En cuanto a talla no se observó diferencia alguna de los dos grupos (Tabla 11). Tomando el parámetro de peso podemos observar que en relación con los pesos menores de 2.000 grs. no hubo diferencia alguna pero ya en relación con los pesos mayores de 3.000 grs. encontramos que si hay una franca diferencia $(\mathrm{P}<.0022)$, lo que nos confirma lo sostenido por otros autores que afirman el menor peso de los neonatos de las adolescentes. En relación con las malformaciones fetales no se observó diferencia en los grupos (Tabla 13).

Si analizamos las complicaciones del puerperio inmediato no observamos ninguna complicación grave en ninguno de los grupos, las lesiones del periné, desgarros de $\mathrm{G}$ 20. y G 3o. fueron más frecuentes en los controles que en las adolescentes, sin significación estadística.

En cuanto a las complicaciones del neonato, se analizaron los siguientes parámetros: Prematuridad, síndrome de dificultad respiratoria, infección y hemorragia cerebral, no se observó diferencia estadísticamente significativa (Tabla 15).

En relación con las complicaciones del puerperio, a los 8 días, se analizan los siguientes parámetros; infección genital, infección de vías urinarias, dehiscencia de suturas y otras, encontrándose que éstas fueron mayores en los controles, sobre todo en lo que se refiere a la infección $(\mathrm{P}<.0045)$ (ver Tabla 16). 


\section{BIBLIOGRAFIA}

1. Ahued JR., Lawenberg E., Vargas G. et al. Atención Obstétrica en la adolescente. VIII Congreso Mundial de Obstetricia y Ginecología, Méx. 1976.

2. Aubry R., and Nesbitt R. High risk obstetrics. II Value semiobjective granding system in identifying the vulnerable group. Am. J. Obstet. and Gynecol. 1969; 103: 972.

3. Barry Z., Joel A. et al. Neonatal autcome: Is adolescent pregnancy arisk factor? Pediatrics 1983; 71: 489.

4. Barrios J., Ramos A. Aspectos perinatales de la adolescente. Rev. Col. Obstet. Ginecol. 1985; 36: 102.

5. Davidson Erza JR. An analysis of adolescent Healt care and the role of the Obtetrician Gynecologist. Am. J. Obstet. and Gynecol. 1981; 139: 845.

6. Boniie WC. et al. Infants of adolescent mothers. Am. J. Dis. C. 1984; 243.

7. Duarte A. Embarazo en la adolescente es siempre de alto riesgo (?). Rev. Col. Obstet. Ginecol. 1975; 26: 385.

8. Duarte A. Embarazo en adolescentes de 11 a 15 años. Rev. Col. Obstet. Ginecol. 1973: 24: 259.

9. Castellanos R. et al. Problemas de las adolescentes de 15 a 16 años de edad. Rev. Venezolana Obstet. Ginecol. 1972; 32: 25.5.

10. Gómez JA. Obstetricia en adolescentes. Rev. Col. Obstet. Ginecol. 1972; 23: 353 .

11. Herrera M. Embarazo en la adolescente Ginecol. Obstet. Méx. 1980; $59: 59$.

12. Guerrero A. et al. El riesgo del embarazo en la paciente adolescente. Ginecol. Obstet. Méx. 1975; 37: 119.

13. Lamers William JR. Problemas que presentan las adolescentes en Obstetricia Obstet. Ginecol. 1970; 727.
14. Mc Ganety WJ. et al. Pregnancy in the adolescent. Am. J. 1963; 103: 773.

15. Ruiz V., Pedraza M. Gestación en la adolescente. Ginecol. Obstet. Méx. 1974; 35: 117 .

16. Ruiz JA. et al. Problemas obstétricos de la adolescente. Ginecol. Obstet. Méx. 1976; 40: 337.

17. Russell JR. Actividad sexual y sus consecuencias en las mujeres de 13 a 19 años. Ginecol. Obstet. Temas Actuales 1974; 683.

18. Ryam JR., Schneider J. Complicaciones Obstétricas en las adolescentes. Clin. Obstet. Ginecol. 1978; 4: 1243.

19. Santigomez G., Pérez JL. et al. Embarazo y parto en las adolescentes. VIII Congreso Mundial Obstet. Ginecol. Méx. 1975.

20. Standy M., Audrey SP. Caracteristics of the Mother and Child in Teenage Pregnancy. Am. J. Obstet. Gynecol. 1983; 137: 365.

21. Szzedrin W. Estudio de la embarazada adolescente. Rev. Obstet. Ginecol. Venezuela, 1975; 35: 25.

22. Tobón G. et al. Materia de consulta más frecuente 620 adolescentes. Rev. Col. Obstet. Ginecol. 1977; 23: 399.

23. Tyrer M., Audrey SP. Cómo cubrir las necesidades especiales de las adolescentes embarazadas. Clin. Obstet. Ginecol. Nort. Am. 1977; 663.

24. Uriza D. Embarazo en adolescentes. Rev. Col. Obstet. Ginecol. 1983; 34: 102 .

25. Sally D. Embarazo en las adolescentes. Cli. Pediatrics Nort. Am. 1989; 3: 715 .

26. Makinson C. The Healt Consequences of Taenage fertility. Fam. Plann pers pect $1985 ; 17: 132$. 